\section{Dip Pen Nanolithography: A Desktop Nanofabrication Approach Using High- Throughput Flexible Nanopatterning}

\author{
Jason Haaheim and Omkar A. Nafday \\ NanoInk Inc. \\ jhaaheim@NANOINK.net
}

\section{Introduction}

Dip Pen Nanolithography (DPN) is a scanning probe lithography technique where an atomic force microscope tip is used to transfer molecules to a surface via a solvent meniscus. This technique allows surface patterning on scales of under 100 nanometres. DPN is the nanotechnology analog of the dip pen (also called the quill pen), where the tip of an atomic force microscope cantilever acts as a "pen," which is coated with a chemical compound or mixture acting as an "ink," and put in contact with a substrate, the "paper."

DPN enables direct deposition of nanoscale materials onto a substrate in a flexible manner. The vehicle for deposition can include pyramidal scanning probe microscope tips, hollow tips, and even tips on thermally actuated cantilevers. Recent advances have demonstrated massively parallel patterning using two-dimensional arrays of 55,000 tips, depicted below. Applications of this technology currently range through chemistry, materials science, and the life sciences, and include such work as ultra high density biological nanoarrays, additive photomask repair, and brand protection for pharmaceuticals.

The transfer of a molecular 'ink' from a coated AFM tip to a substrate was first reported by Jaschke and Butt in 1995 [1]. The technique was further developed by a research group at Northwestern University led by Chad Mirkin [2] who also introduced the term "DPN". The company NanoInk, Inc. holds a patent on Dip Pen Nanolithography, and "DPN" and "Dip Pen Nanolithography" are trademarks or registered trademarks of NanoInk [3].

Sinceitsinception tenyearsago, Dip Pen Nanolithography ${ }^{\circ}\left(\mathrm{DPN}^{\star}\right)$ has gone from a single-ink, single-tip research technique, to a multiink, multi-tip, versatile surface patterning system. In some cases,
DPN is capable of exceeding the throughput of e-beam lithography $\left(10^{8} \mu \mathrm{m}^{2} / \mathrm{hr}\right)$.

As DPN has evolved into a high-throughput patterning method, NanoInk has made numerous advances in the DPN toolkit, both in terms of research and patterning technology. It has also developed the concept of desktop nanofabrication - an approach that allows a non-expert atomic force microscope (AFM) user to create high resolution, scalable nanostructures rapidly with a wide variety of materials, drawing upon well-characterized ink and substrate pairings. Furthermore, it demonstrates the viability of DPN for high-throughput nanofabrication, by offering massively parallel nanopatterning capabilities with its 55,000 tip 2D nano PrintArray $^{\mathrm{TM}}$.

\section{Attributes of DPN}

DPN is a proven nanopatterning technique that offers solutions to the complex obstacles inherent in creating architecture at the nanoscale. Whether that architecture is physical or chemical, there are principally two approaches to building nanostructures top-down or bottom-up.

DPN uses the AFM tip to deposit molecules by direct-write on suitable substrates, enabling both approaches to nanofabrication. Precision nanoscale deposition is a fundamental requirement for much current nanoscience research, and depositing a variety of materials as nanoscale features onto diverse surfaces is a challenging requirement for nanoscale processing systems. DPN is an inherently additive Scanning Probe Microscope (SPM) based technique that operates under ambient conditions, making it suitable to deposit a wide range of biological, organic, and inorganic materials, at specified locations.

Table 1: A comparison of nanopatterning techniques; DPN's competitive advantages relative to other methods. $276 \times 100 \mathrm{~mm}$

Table 1 provides an instructive look at DPN's place among nanopatterning techniques: it is highly scalable with the use of multi-pen arrays; it is a technique that enables both bottom-up nanofabrication (e.g., self-assembly, templating) and top down

Table 1: Comparing Nanopatterning Techniques - DPN's Competitive Advantages

\begin{tabular}{|c|c|c|c|c|c|c|c|c|c|}
\hline \multirow{2}{*}{ Approach } & \multirow{2}{*}{$\begin{array}{c}\text { Nanopatter- } \\
\text { ning } \\
\text { Technique }\end{array}$} & \multirow{2}{*}{$\begin{array}{l}\text { Serial / } \\
\text { Parallel }\end{array}$} & \multirow{2}{*}{$\begin{array}{c}\text { Material } \\
\text { Flexibility }\end{array}$} & \multirow{2}{*}{$\begin{array}{c}\text { Litho } \\
\text { Resolution }\end{array}$} & \multirow[b]{2}{*}{ Litho Speed } & \multirow{2}{*}{$\begin{array}{c}\text { Registration } \\
\text { Accuracy }\end{array}$} & \multirow[b]{2}{*}{ Cycle Time } & \multicolumn{2}{|c|}{ Cost } \\
\hline & & & & & & & & Purchase & Operation \\
\hline \multirow{3}{*}{ Top down } & $\begin{array}{c}\text { Photo- } \\
\text { lithography }\end{array}$ & parallel & no & $\sim 35 \mathrm{~nm}$ & very fast & high & Weeks & $>\$ 10 \mathrm{M}$ & high - masks \\
\hline & $\begin{array}{c}\text { E-Beam } \\
\text { Lithography }\end{array}$ & serial & no & $\sim 15 \mathrm{~nm}$ & medium & high & Days & $>\$ 1 \mathrm{M}$ & high \\
\hline & $\begin{array}{c}\text { Nanoimprint } \\
\text { Lithography } \\
\text { (NIL) }\end{array}$ & parallel & no & $\sim 10 \mathrm{~nm}$ & fast & high & days-week & $>\$ 500 \mathrm{k}$ & $\begin{array}{l}\text { moderate - } \\
\text { molds }\end{array}$ \\
\hline Enables Both & $\begin{array}{c}\text { Dip Pen } \\
\text { Nano- } \\
\text { lithography } \\
\text { (DPN) }\end{array}$ & $\begin{array}{c}\text { serial or } \\
\text { parallel pens }\end{array}$ & yes & $14 \mathrm{~nm}$ & $\begin{array}{l}\text { highly } \\
\text { scalable; } \\
\text { 2D speeds } \\
\text { exceeds } \\
\text { e-beam }\end{array}$ & $\begin{array}{l}\text { extremely } \\
\text { high }\end{array}$ & $\begin{array}{l}\text { hours - } \\
\text { change on } \\
\text { the fly }\end{array}$ & $<\$ 350 \mathrm{k}$ & low \\
\hline \multirow{2}{*}{ Bottom up } & $\begin{array}{c}\text { Microcontact } \\
\text { Printing } \\
(\mu \mathrm{CP})\end{array}$ & parallel & yes & $\sim 100 \mathrm{~nm}$ & fast & low & days-week & $\sim \$ 200 \mathrm{k}$ & $\begin{array}{l}\text { moderate - } \\
\text { masks }\end{array}$ \\
\hline & $\begin{array}{l}\text { Scanning } \\
\text { Tunneling } \\
\text { Microscopy }\end{array}$ & serial & limited & atomic & very slow & $\begin{array}{l}\text { extremely } \\
\text { high }\end{array}$ & Days & $>\$ 250 \mathrm{k}$ & low \\
\hline
\end{tabular}




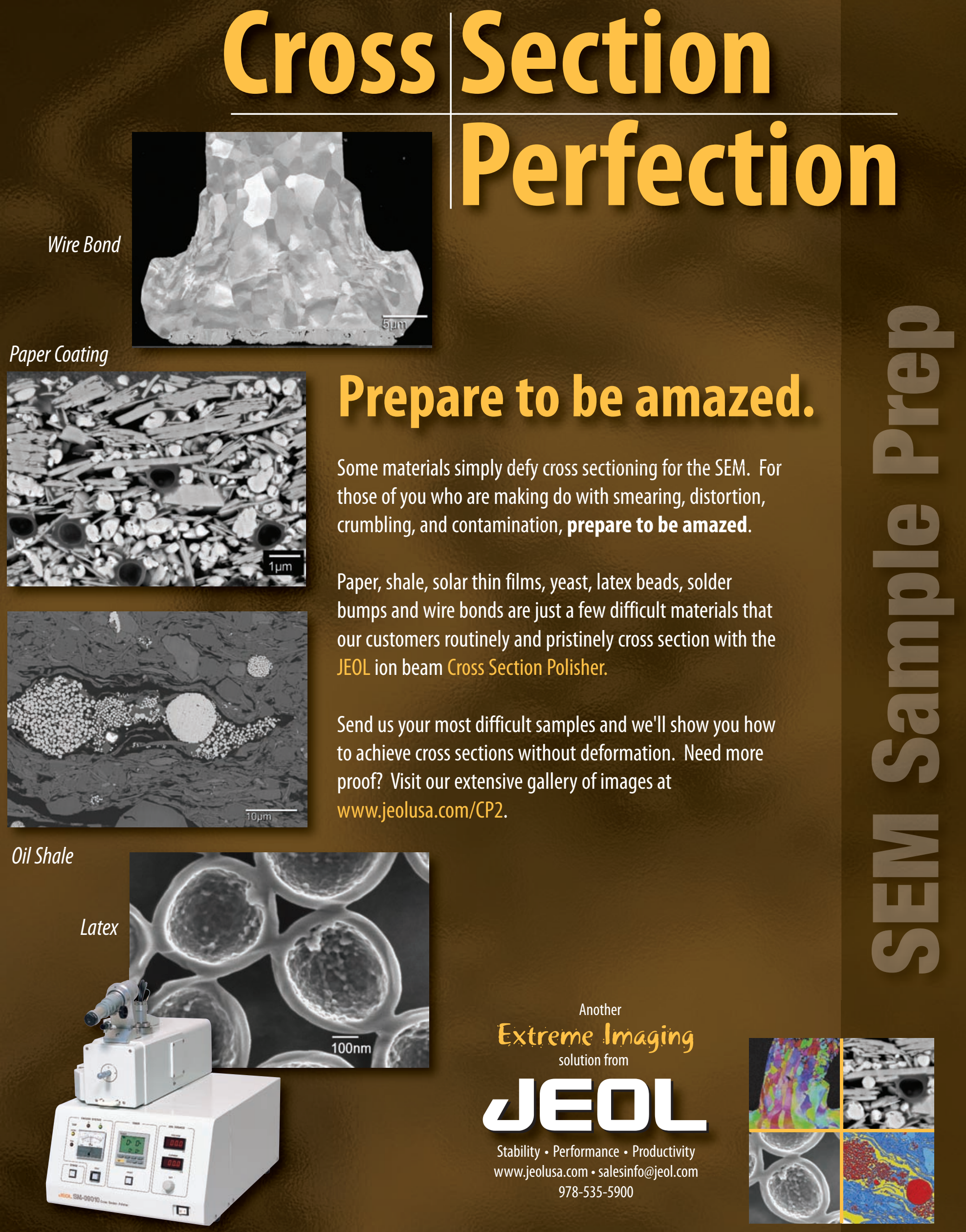



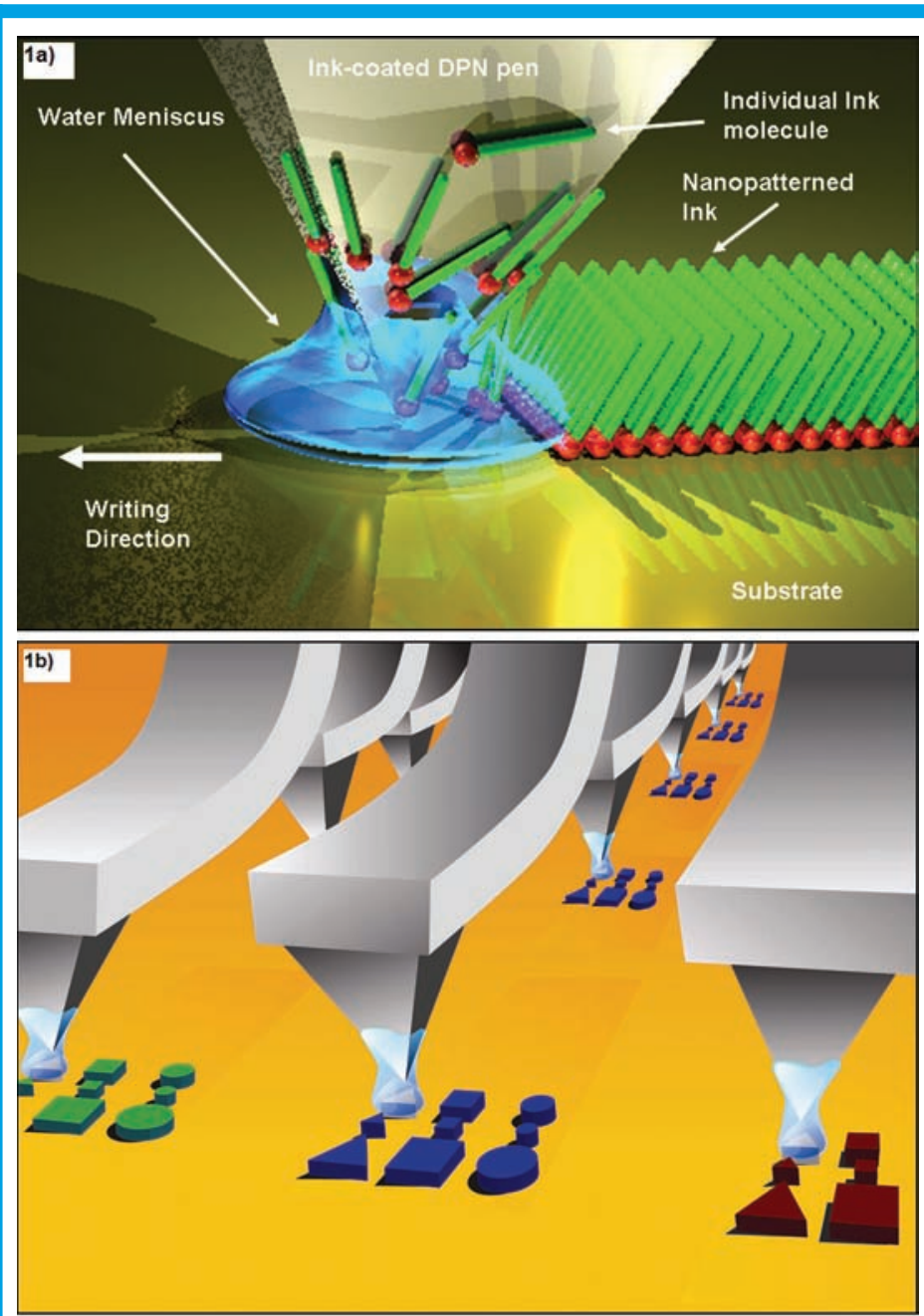

Figure 1a: Schematic of the Dip Pen Nanolithography (DPN) process. A molecule-coated single AFM tip deposits ink via a water meniscus onto a substrate. Reproduced with permission from Nature Publishing Group (Salaita et al., 2007). Figure 1: b) Schematic representation of the DPN process scaled up for massively parallel nanopatterning. The graphic depicts the ultimate aim of rapidly creating a variety of structures on the fly, with different inks on each tip.

fabrication via etch resist-based inks and it is high resolution (14 $\mathrm{nm}$ line widths, $20 \mathrm{~nm}$ pitches).

\section{Ink Transport in DPN - Transferring Molecules from Tip to Substrate}

In its simplest terms, a successful ink system transfers molecules from a probe tip to a substrate. The AFM tip is coated in ink which then moves onto the substrate by capillary transport through the meniscus. (Figure 1a)

For a more powerful transfer, the ink can be designed to react with the surface to be patterned and the resulting chemisorption enhances the movement of the ink from the AFM tip through the water meniscus to the substrate.

NanoInk's DPN $5000^{\mathrm{mm}}$, the latest instrument in the popular NSCRIPTOR family of DPN systems, is based on scanning probe microscopy (SPM) technology and is capable of both pattern fabrication and immediate verification of the result by AFM imaging. It allows precise control of the environmental conditions important for a typical DPN experiment, using an environmental chamber capable of controlling temperature and $\mathrm{RH}(0-75 \%)$ through a real-time feedback loop.

\section{Ink Delivery Systems}

The process of inking a DPN pen (i.e., an AFM probe with ink on it) can be as trivial as dipping it in a vial with a pair of tweezers. However, repeatability and process control are cornerstone elements of DPN applicability - where DPN serves as a means to an end - and there are two critical components of inking that must be addressed to achieve this overall process control:

- Selectively delivered ink, with no cross-contamination;

- Uniform cantilever loading.

To ensure this, NanoInk has developed complementary tools - Universal InkwellsTM and DNA InkwellsTM - to enable controllable inking by aligning microfluidic channels precisely with the cantilever arrays, keeping in mind the cantilever-to-cantilever spacing. Inkwells uniquely provide isolation, containment and optimized fluid flow (to the microwell, and not vice versa) and these devices represent the next generation of inkwell development.

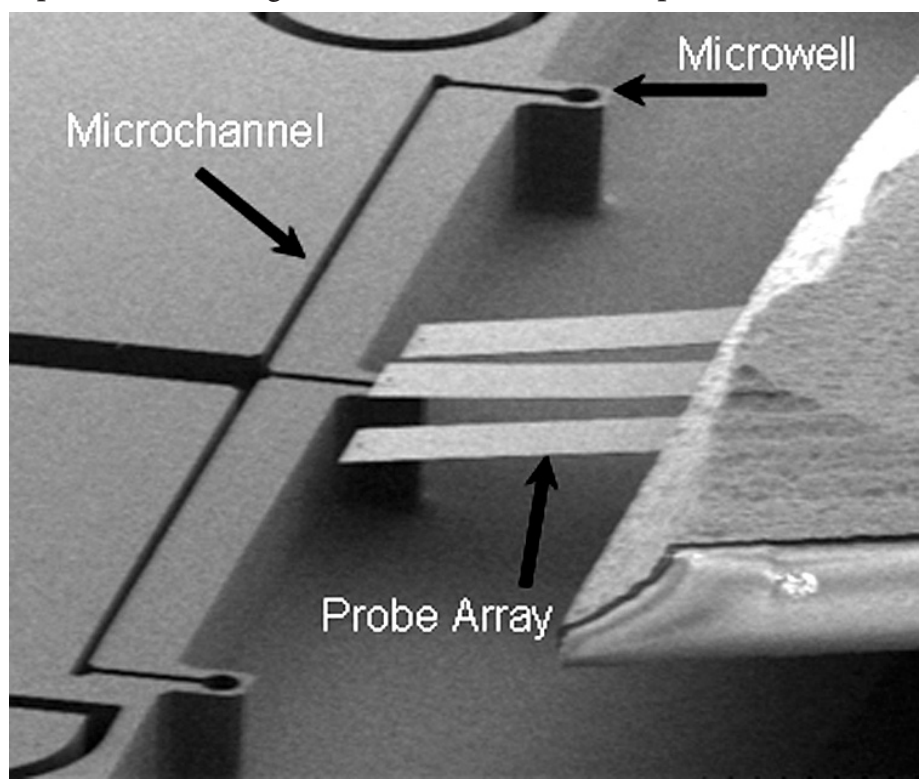

Figure 2: Example of NanoInk's Inkwell technology for the supply of inks to the "pens" through MEMs fabricated devices chemically treated so inks are kept in their own well resulting in no cross-contamination.

\section{Actuated Cantilevers}

DPN patterns that are simply written and imaged with a single inked tip can contaminate the pattern even as it is imaged, depending on lithography conditions. This contamination can lead to reduced phase contrast with continued imaging, and eventual pattern overwriting.

Selective inking and individual cantilever actuation becomes even more important for the patterning of biological inks, where cross-contamination can lead to non-specific binding, or interfere with later fluorescence characterization.

The Active PenTM tool has been specifically engineered for flexible and multiplexed nanopatterning, with lateral tip spacing as low as $23 \mu \mathrm{m}$. Using thermal bimorph technology, individual cantilevers can be actuated to enable multi-ink writing without any cross-contamination or unintended surface patterning. Additionally, reader tips can be left clean to image surface patterns, or address specific surface structures without unintentional inking.

As combined patterning of biological materials, small functional molecules and inorganic nanoparticles becomes increasingly necessary to produce functional nanostructures, such tools will be 
pivotal to nanotechnology research and development.

\section{Commercially Available Massively Parallel Nanopatterning}

Massively parallel two-dimensional nanopatterning with DPN is now commercially available via NanoInk's 2D Nano PrintArray, making DPN a high-throughput, flexible and versatile method for precision nanoscale formation. (Figure $1 \mathrm{~b}$ )

Now, with a 2D array of 55,000 pens on a centimetre square probe chip, the highest cantilever density ever reported, throughput exceeding $1 \times 107 \mu \mathrm{m} 2 / \mathrm{hr}$ and a dot size standard deviation of only $16 \%$, has been achieved.

Fundamentally, this enables flexible direct-writing with a variety of molecules, simultaneously generating 55,000 duplicates at the resolution of single-pen DPN. It is the only way to flexibly pattern a variety of materials with nanoscale resolution $(<80 \mathrm{~nm})$ across $\mathrm{cm} 2$ areas.

2D DPN templating and biomolecule deposition opens up a completely new area of single particle biology; it is possible to probe interactions between surfaces and single viruses, spores or cells.

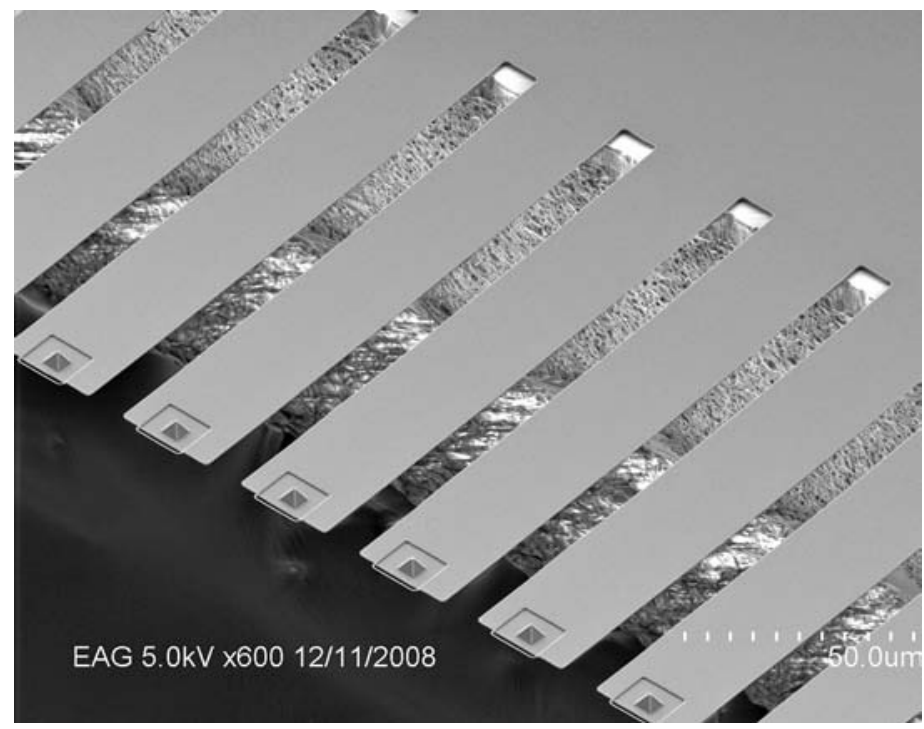

Figure 3: $1 D$ array of pens for easily controlled parallel writing of nanoscale features.

\section{The Desktop Nanofab Approach}

The concept of desktop nanofabrication allows a non-expert user to rapidly create high resolution, scalable nanostructures with a wide variety of materials, drawing upon well-characterized ink and substrate pairings. In order to get uniform feature sizes and reliable tip inking in DPN, the development of a suitable ink carrier material is pertinent.

Beyond DNA, NanoInk is currently pursuing several tracks for the different classes of universal inks. Suitable carriers for biological ink DPN include Agarose, PEG, phospholipids and possibly gelatine nanoparticles.

\section{Summary}

Since its discovery, DPN has proved capable of patterning a variety of materials onto a variety of substrates. DPN-deposited patterns have been used as a resist in an etching step, or as a template for bottom up assembly. As DPN works in ambient conditions, it offers a significant advantage for materials that are environmentally sensitive or incompatible with existing microfabrication techniques, such as biomolecules, conductive polymers, or ceramics (e.g. for sensors and molecular electronics).
The DPN toolkit interfaces the inorganic with the organic, the world of microelectronics with the world of biochemistry and polymers. All of these attributes have become highly scalable with our development of a commercial 2D nanopatterning solution - one that is easy to use and readily implemented on any NSCRIPTOR. With the 2D nano PrintArray, NanoInk is advancing DPN as a technique for high-throughput nanopatterning.

With such technology now proven and in practice, desirable future developments could include laser feedback on viewable cantilevers for immediate imaging, and automated step-and-repeat lithographic routines.

Multiplexed ink delivery to a large number of tips remains a fundamental challenge - one we are approaching through a variety of methods, including InkTroughTM channels and different vapor coating techniques. Such a capability would enable multiplexed combinatorial libraries of nanoscale patterns across large areas.

\section{References}

1. Jaschke M.; Butt, H.-J. "Deposition of Organic Material by the Tip of a Scanning Force Microscope" Langmuir, 1995, 11, 1061-1064.

2. Piner, R. D.; Zhu, J.; Xu, F.; Hong, S.; Mirkin, C. A. "Dip Pen Nanolithography," Science, 1999, 283, 661-663.

3. The first three paragraphs, in italics, were written by Jason Haaheim and other Wikipedia authors. Reference: "Dip-Pen Nanolithography." Wikipedia, The Free Encyclopedia. 29 Dec 2008, 23:14 UTC. 15 Feb 2009 <http://en.wikipedia.org/w/ index.php?title=Dip-Pen_Nanolithography\&oldid=260766326>

\section{ASSISTANT-ASSOCIATE PROFESSOR MATERIALS SCIENCE-ELECTRON MICROSCOPY SCHOOL OF MATERIALS, ARIZONA STATE UNIVERSITY}

The interdisciplinary School of Materials is a large academic unit with both graduate and undergraduate degree programs, and well established research programs in electronic and energy materials, as well as in other diverse areas of materials science. The School is expanding its well established microscopy activities to include aberration corrected high resolution analytical transmission electron microscopy. Announcement of this tenure track position, at the Associate or Assistant Professor level, coincides with acquisition of a new aberration corrected electron microscope to be installed in the LeRoy Eyring Center for Solid State Science/John Cowley Center for High Resolution Electron Microscopy. The successful applicant is expected to be a primary user of this new instrument for his/her personal externally funded research program, and to take a leadership role in familiarizing research personnel with aberration corrected electron microscopy. Applications will be reviewed beginning March 2, 2009; if not filled, the 15th and 30th of each month thereafter until the search is closed. Additional information is available at http://som.asu.edu/employment/index.php.

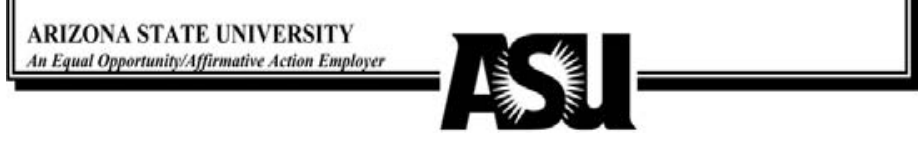

